\title{
Asociación entre factores socioeconómicos y el índice de masa corporal en preescolares de bajos ingresos de una institución educativa de Floridablanca, Colombia
}

\author{
Association between socioeconomic factors and body mass \\ index among low-income preschoolers from an educational \\ institution of Floridablanca, Colombia
}

\section{ABSTRACT}

We determined the association between socioeconomic factors and body mass index z-score (BMIZ) in preschoolers from the Colombo-German Foundation from Floridablanca, Colombia. Height and weight were measured in a random sample of 112 preschool children aged 2-5 years of age; their parents or caregiver answered a survey. The multiple generalized linear models showed in the whole sample that BMIZ significantly decreased with household's socioeconomic strata 1 and 2 compared with strata zero, and in high-income families. In girls, BM IZ significantly increased when another family member, neighbor, or friend was responsible for deciding about feeding on weekdays. In boys, $\mathrm{BM} I \mathrm{Z}$ increased when they ate alone and when their grandmother was responsible for preparing food on weekdays. It is concluded that socioeconomic factors influencing BM IZ are similar to those that influence health inequity.

Key words: Socioeconomic factors, risk factors, body mass index, pediatric obesity.

\section{INTRODUCCIÓN}

Los cambios en el patrón de fertilidad, mortalidad y los comportamientos relacionados con la dieta y la actividad física han contribuido al incremento de las enfermedades crónicas no transmisibles (ENT) como obesidad, enfermedades cardiovasculares (ECV) y cáncer (1-3). La obesidad infantil es considerada un problema de salud pública mundial que va en aumento. Ng y Cols., encontraron que la prevalencia de exceso de peso entre 1980 y 2013 en niños y adolescentes (menores de 20 años) de países en desarrollo pasó de $8,1 \%$ a $12,9 \%$ en varones y de $8,4 \%$ a $13,4 \%$ en mujeres (3). En la Región de las Américas en niños de 0 a 5 años de la República Dominicana pasó de 2,2\% en 1991 a $5,6 \%$ en el 2007; en el Salvador de 1,5\% en 1993 a 3\% en el 2008, en el Perú de 2,5\% en 1992 a 3,2\% en el 2012. En los niños de 0 a 4 años del Caribe oriental de 7,4\% en 2000 a $14,8 \%$ en el 2010 (4) y en Colombia de $4.9 \%$ en 2005 a $5.2 \%$ en el 2010 (5).

La obesidad infantil es un problema preocupante por las consecuencias sobre la salud física y mental (6) lo que aumenta rápidamente en población es de bajos ingresos ( 1 , 2). El costo de su tratamiento impone una carga económica
(1) Observatorio Epidemiológico de Enfermedades Cardiovasculares. Centro de Investigaciones Epidemiológicas. Universidad Industrial de Santander, Bucaramanga, Colombia. (2) Escuela de Nutrición y Dietética. Universidad Industrial de Santander. Bucaramanga. Colombia. (3) Fundación Colombo Alemana Volver a Sonreir Floridablanca, Colombia.

Dirigir la correspondencia a: Profesora

Gloria Esperanza Prada Gómez Centro de Investigaciones Epidemiológicas Facultad de Salud, U niversidad Industrial de Santander Carrera 32 No. 29-31, tercer piso, Bucaramanga, Colombia E-mail: pradauis@gmail.com / gprada@uis.edu.co Teléfax: (57-7) 6323215

Este trabajo fue recibido el 16 de Marzo de 2015 y aceptado para ser publicado el 10 de Agosto de 2015.

insostenible para los países (2), se requieren estudios para entender como influyen la pobreza, los factores socioculturales y las variables del hogar en su incremento $(7,8)$.

Al respecto, se ha evidenciado una asociación del exceso de peso con factores sociales y económicos como la edad de la madre, educación de los padres, estrato social, y ambiente alimentario (9). Investigaciones realizadas en los últimos 15 años, mostraron una relación inversa entre el nivel socioeconómico de la familia (NSE) y la obesidad en niños (7), en España en 6.139 niños de 2-15 años de edad se encontró esta asociación (10), McDonald y Cols. estudiaron en Colombia 3075 niños entre 5 y 12 años y encontraron asociación positiva entre el estrato socioeconómico y exceso de peso en el análisis bivariado, no así en el análisis multivariado (11).

La relación NSE y exceso de peso en preescolares en Colombia ha sido poco explorada y menos en población de estrato socioeconómico bajo.

El objetivo de este trabajo fue establecer los factores socioeconómicos asociados al Índice de Masa Corporal (IMC) en niños y niñas usuarios de la Fundación Colombo-Alemana y localizados en el municipio de Floridablanca, Santander, Colombia. 


\section{SUJETOS Y MÉTODOS}

Estudio de corte transversal realizado durante los años 2013 y 2014. El universo estuvo constituido por 335 niños y niñas de dos a cinco años, que asistían de lunes a viernes de 8:00 a las 14:00 a la Fundación Colombo-Alemana; institución sin fines de lucro, creada para mejorar la calidad de vida y el fortalecimiento familiar de las comunidades en situación de vulnerabilidad del municipio de Floridablanca, el cual cuenta con una población estimada para el 2012 de 271,728 habitantes.

Se calculó un tamaño muestral de 128 preescolares, considerando un nivel de confianza del $95 \%$, poder de $80 \%$, una precisión del 5,0\% y una prevalencia de exceso de peso esperada de $16,0 \%$ (12). Al tener en cuenta $32,8 \%$ de pérdidas, se seleccionaron de manera aleatoria 170 menores, de los cuales 112 de sus representantes legales firmaron el consentimiento informado y aceptaron participar, siendo la tasa de participación del 66\%. La selección de la muestra se realizó mediante muestreo aleatorio simple a partir del listado de preescolares.

Previo a la recolección se socializó el proyecto de información en reuniones de padres de familia. Los padres o representantes de los niños y niñas seleccionados junto con la persona encargada de suministrar y preparar la alimentación al menor fueron citados a las instalaciones de la Fundación, los que no asistieron fueron abordados en su hogar.

Siete estudiantes del programa de Nutrición y Dietética de la Universidad Industrial de Santander y un nutricionista, entrenados en métodos de entrevista directa y mediciones antropométricas aplicaron la encuesta que indagaba por factores biológicos, económicos, sociodemográficos del niño(a) y sus familiares, antecedentes patológicos familiares de los padres y parentesco de la persona encargada del cuidado. Se destaca que la estratificación socioeconómica colombiana, es una clasificación de los domicilios o viviendas a partir de sus características físicas y de su entorno, en seis grupos o estratos: 1. Bajo-bajo, 2. Bajo, 3. Medio-bajo, 4. Medio, 5. Medio-alto, 6. Alto. Las personas víctimas del conflicto armando por desplazamiento son consideradas en estrato cero. La tasa de ocupación se calculó dividiendo el número de personas ocupadas sobre la población en edad de trabajar, constituida por todas las personas de 12 años en adelante. En los ingresos familiares se utilizó como referencia el salario mínimo legal vigente (SMLV) para Colombia en el año 2013, que correspondió a 589500 pesos colombianos (332 dólares USD).

Se realizaron mediciones de peso y talla siguiendo las recomendaciones de la ISAK (International Standards for Anthropometrics Assessment) (13) y se utilizaron básculas digitales marca Tanita HD-314 con capacidad Máxima 150 $\mathrm{kg}$, con calibración automática $\mathrm{d}=0.1 \mathrm{~kg}$, tallímetros marca SECA Modelo 206.

La prevalencia de obesidad y sobrepeso se calculó teniendo en cuenta la clasificación de la Organización Mundial de la Salud. Así, después de obtener el puntaje z del IMC para la edad y sexo utilizando el programa ANTHRO (14), los preescolares se clasificaron en obesos si el puntaje $z$ estaba por encima de 2 y en sobrepeso si el puntaje $z$ se encontraba entre 1 y 2.

Las variables cualitativas se presentaron en frecuencias absolutas y relativas y sus respectivos intervalos de confianza del 95\%; en las variables cuantitativas se calculó la media aritmética y la desviación estándar o la mediana y el rango intercuartílico (percentil 25 - percentil 75) según la distribución de la variable evaluada con la prueba de Shapiro-Wilk. En el análisis bivariado se utilizaron modelos lineales generalizados (MLG) simples teniendo en cuenta el puntaje z del IMC para la edad y sexo como variable dependiente. Se aplicaron MLG múltiples con el fin de establecer si existían variables asociadas al puntaje $z$ del IMC para la edad y sexo. Se exploraron en el modelo final aquellas variables que obtuvieron en el bivariado un valor $p<0,20$, aunque se excluyeron las variables del padre: edad, escolaridad y ocupación, por tener 35 datos faltantes. En el modelo se usó una dispersión de dos veces el promedio y el método cuasi-likelihood. El MLG es un modelo semiparamétrico para datos heterogéneos que no cumplen los supuestos de normalidad, adicionalmente por el método cuasi-likelihood no es necesario especificar la distribución de la variable respuesta; la interpretación de los coeficientes es directa y se logra el ajuste de sobredispersión de los datos (15). La evaluación del modelo final se realizó mediante la prueba linktest y se realizó el análisis de residuales deviance. Así mismo se aplicó el modelo a niños y niñas por separado. Los datos fueron digitados en el software EpiData Entry version 2.0 (16) y exportados al software estadístico Stata 13 (17) para su análisis.

Según la Resolución No. 008430 del 04 de octubre de 1993 del Ministerio de Salud de Colombia (18), esta investigación se consideró sin riesgo. Se garantizaron los principios éticos de respeto, autonomía, justicia y confidencialidad. El presente estudio fue aprobado por el Comité de Etica para la Investigación Científica de la Facultad de Salud de la Universidad Industrial de Santander y se solicitó el consentimiento informado por escrito del representante legal de los menores.

\section{RESULTADOS}

La muestra quedó conformada por 55 niñas y 57 niños, de los cuales $54,5 \%$ tenía entre cuatro y cinco años. El puntaje $z$ del IMC para edad y sexo fue significativamente mayor en los niños varones comparado con las niñas $(\beta=0,17)$. (tabla 1).

El $15,2 \%(n=17)[$ IC95\% 9,6-23,2] se encontraba en sobrepeso y $1,8 \%(n=2)$ [IC95\% 0,4-7,0] en obesidad. La mediana del puntaje z del IMC para la edad y sexo fue de 0,41 (mínimo=-3,02; máximo=2,89). El 51,5\% de los participantes cursaba el nivel educativo de párvulos, 28,0\% jardín 1 y 20,5\% jardín 2 .

Respecto a las características de la madre (tabla 2), la mediana de edad fue 27 años (RIC 23-33), 70,5\% estaba en unión libre o casada, 66,7\% estudió hasta secundaria, y el $55,4 \%$ estaba trabajando. En relación con los padres, la mediana de edad fue 32 (RIC 28-37), 69,7\% estaba en unión libre o casado, 33,9\% estudió hasta secundaria y 66,1\% estaba trabajando. Resalta que en estas dos últimas variables no se obtuvo el dato de 35 padres dado que el 31,2\% son familias monoparentales.

Se encontró una relación inversa y significativa entre el puntaje $z$ del IMC de los niños estudiados y la edad de la madre, así, por cada año que aumenta la edad de la madre en promedio el puntaje $z$ disminuye 0,02 ; y con la escolaridad de los padres, los hijos de padres con secundaria tienen más puntaje $z$ que aquellos con padres sin escolaridad o con primaria $(\beta=0,28)$ (tabla 2$)$.

En relación con las características socioeconómicas del hogar, la tasa de ocupación fue en promedio 0,66 0,26, $71,4 \%$ pertenecía a los estratos 1 y 2,53,6\% recibía entre 1 y 3,9 SMLV, 69,8\% procedía del área urbana, 57,4\% no era familia desplazada, $71 \%$ recibía subsidios gubernamentales, 
de Organizaciones no gubernamentales (ONG) o de amigos, el 42,0\% vivía en vivienda arrendada. El número de personas que conformaba el núcleo familiar (incluido el niño) era de 1 a 4 para 54,2\%; el número de personas que vivían con el niño estuvo entre 4 y 9 para el 53,6\%, el número de personas que duerme con el niño estuvo entre 0 y 1 para el $53,6 \%$ y el número de niños menores de 7 años (incluido el niño) fue de uno para el 53,6\% (tabla 3).

En el análisis bivariado se encontró disminución significativa del puntaje $z$ del IMC para la edad y sexo en los

TABLA 1

Características de los niños y niñas de la Fundación Colombo-Alemana asociadas al puntaje z IMC para edad y sexo.

Floridablanca, Colombia, 2013.

\begin{tabular}{|c|c|c|c|c|c|c|c|c|c|c|}
\hline & \multicolumn{6}{|c|}{ Medida de resumen } & \multicolumn{4}{|c|}{ Regresión lineal generalizada } \\
\hline & $\mathrm{n}$ & $\%$ & & & Mediana & P25 & P75 & $\begin{array}{c}\text { Coeficiente } \\
\text { Beta }\end{array}$ & IC95\% & Valor $\mathrm{p}$ \\
\hline \multicolumn{11}{|l|}{ Sexo } \\
\hline Femenino & 55 & 49,1 & 39,8 & 58,4 & 0,31 & $-0,14$ & 0,81 & & & \\
\hline \multirow{2}{*}{\multicolumn{11}{|c|}{ Edad }} \\
\hline & & & & & & & & & & \\
\hline 2-3 años & 51 & 45,5 & 36,3 & 54,8 & 0,42 & $-0,22$ & 0,92 & & & \\
\hline 4-5 años & 61 & 54,5 & 45,2 & 63,7 & 0,4 & $-0,09$ & 0,89 & 0,02 & $-0,15$ & $0,18 \quad 0,840$ \\
\hline
\end{tabular}

IMC= Indice de Masa Corporal. P25= Percentil 25. P75= Percentil 75. n=frecuencia absoluta. \%=Porcentaje. IC95\% Intervalo de confianza del $95 \%$.

TABLA 2

Características de las madres y los padres de los niños y niñas de la Fundación Colombo-Alemana asociadas al puntaje $z$ IMC para edad y sexo. Floridablanca, Colombia, 2013.

\begin{tabular}{|c|c|c|c|c|c|c|c|c|c|c|c|}
\hline \multirow[t]{2}{*}{ Variable } & \multicolumn{2}{|c|}{$\begin{array}{l}\text { Medida de } \\
\text { resumen }^{1}\end{array}$} & \multicolumn{2}{|c|}{ IC95\% } & \multicolumn{3}{|c|}{$\begin{array}{l}\text { Puntaje } z \text { del IMC } \\
\text { para edad y sexo }\end{array}$} & \multicolumn{4}{|c|}{ Regresión lineal generalizada } \\
\hline & & & & & Mediana & P25 & P75 & $\begin{array}{l}\text { Coeficiente } \\
\text { Beta }\end{array}$ & & & Valor $\mathrm{p}$ \\
\hline \multicolumn{12}{|l|}{ Características de la madre } \\
\hline $\begin{array}{l}\text { Edad (años)2 } \\
\text { Estado civil }\end{array}$ & 27 & $23-33$ & 25,0 & $28,0^{3}$ & - & - & - & $-0,02$ & $-0,03$ & 0,00 & 0,028 \\
\hline $\begin{array}{l}\text { Union libre/casada } \\
\text { Separada/Divorciada/ }\end{array}$ & 79 & 70,5 & 62,1 & 79 & 0,44 & $-0,15$ & 0,91 & & & & \\
\hline $\begin{array}{l}\text { viuda/Madre Soltera } \\
\text { Escolaridad }\end{array}$ & 33 & 29,5 & 21,0 & 37,9 & 0,31 & $-0,16$ & 0,89 & $-0,12$ & $-0,31$ & 0,06 & 0,182 \\
\hline $\begin{array}{l}\text { Ninguna/Primaria/ } \\
\text { Secundaria }\end{array}$ & 28 & 26,7 & 17 & 33 & 0,30 & $-0,31$ & 0,99 & & & & \\
\hline $\begin{array}{l}\text { Técnica/Tecnóloga } \\
\text { Ocupación }\end{array}$ & 77 & 73,3 & 60,2 & 77,3 & 0,47 & $-0,13$ & 0,91 & 0,09 & $-0,10$ & 0,29 & 0,355 \\
\hline Trabajando & 62 & 55,4 & 46,2 & 64,6 & 0,29 & $-0,13$ & 0,89 & & & & \\
\hline $\begin{array}{l}\text { del hogar } \\
\text { Sin registro }\end{array}$ & 38 & 33,9 & 25,2 & 42,7 & 0,50 & $-0,22$ & 0,85 & 0,04 & $-0,14$ & 0,23 & 0,632 \\
\hline Sin registro & 12 & 10,7 & 5,0 & 16,4 & 0,50 & $-0,22$ & 0,85 & 0,09 & $-0,18$ & 0,37 & 0,510 \\
\hline \multicolumn{12}{|l|}{ Características del padre } \\
\hline $\begin{array}{l}\text { Edad (años) }{ }^{4} \\
\text { Estado civil }\end{array}$ & 32 & $28-37$ & 30,0 & 34,03 & - & - & - & $-0,01$ & $-0,02$ & 0,00 & 0,087 \\
\hline $\begin{array}{l}\text { Union libre/Casado } \\
\text { Separado/Divorciado/ }\end{array}$ & 76 & 69,7 & 59,2 & 76,5 & 0,415 & $-0,255$ & 0,89 & & & & \\
\hline $\begin{array}{l}\text { viudo/Padre Soltero } \\
\text { Escolaridad }\end{array}$ & 33 & 30,3 & 21,0 & 37,9 & 0,4 & $-0,13$ & 0,92 & 0,09 & $-0,08$ & 0,26 & 0,319 \\
\hline Ninguno-Primaria & 34 & 30,4 & 21,8 & 38,9 & 0,19 & $-0,35$ & 0,81 & & & & \\
\hline $\begin{array}{l}\text { Secundaria } \\
\text { Técnico/Tecnólogo/ }\end{array}$ & 38 & 33,9 & 25,2 & 42,7 & 0,19 & $-0,35$ & 0,81 & 0,28 & 0,07 & 0,48 & 0,009 \\
\hline Uni-versitario & 5 & 4,5 & 0,6 & 8,3 & 0,46 & 0,02 & 0,91 & $-0,08$ & $-0,50$ & 0,34 & 0,718 \\
\hline Sin dato & 35 & 31,2 & 22,7 & 39,8 & $-0,09$ & $-0,15$ & 0,13 & 0,15 & $-0,07$ & 0,36 & 0,175 \\
\hline \multicolumn{12}{|l|}{ Ocupación } \\
\hline $\begin{array}{l}\text { Trabajando } \\
\text { Buscando trabajo/ }\end{array}$ & 74 & 66,1 & 57,3 & 74,8 & 0,31 & $-0,16$ & 0,88 & & & & \\
\hline Estudiando & 3 & 2,7 & 0,0 & 5,7 & 0,68 & 0,54 & 0,91 & 0,39 & $-0,12$ & 0,91 & 0,136 \\
\hline Sin registro & 35 & 31,2 & 22,7 & 39,8 & 0,68 & 0,54 & 0,91 & 0,03 & $-0,15$ & 0,21 & 0,738 \\
\hline
\end{tabular}

IMC= Indice de Masa Corporal. P25= Percentil 25. P75= Percentil 75. IC95\% Intervalo de confianza del 95\%. - No aplica para variables cuantitativas.

${ }^{1}$ Mediana y Rango intercuartílico para la variable edad, frecuencia absoluta $(\mathrm{n})$ y relativa (\%) en las demás variables. ${ }^{2} \mathrm{n}=105 .{ }^{3}$ Intervalo de confianza del $95 \%$ para la mediana. ${ }^{4} n=77$. 
preescolares que pertenecían a hogares de estratos 1 y 2 $(\beta=-0,38)$, con ingresos familiares entre 0,6 y $1,0 \mathrm{SMLV}(\beta=-$ $0,46)$ y entre 1,5 y $3,9 \mathrm{SMLV}(\beta=-0,42)$, de procedencia rural $(\beta=-0,22)$, que habitaban una vivienda arrendada $(\beta=-0,32)$ y que contaban con 2 a 4 niños menores de 7 años $(\beta-0,24)$ (tabla 3).

En el análisis bivariado, se encontró un aumento significativo del puntaje $z$ del IMC para edad y sexo con cuidador del niño(a) (abuela: $\beta=0,34$; otro familiar: $\beta=0,30$ ), persona que responde por la preparación de alimentos de lunes a viernes (abuela: $\beta=0,39$; otro familiar: $\beta=0,47$ ) y persona que decide la alimentación de lunes a viernes (abuela/ambos padres: $\beta=0,22$; otro familiar, vecina o amiga: $\beta=0,40$ ) (tabla 4 ).

Las madres fueron quienes principalmente respondieron la encuesta $(76,8 \%)$, cuidaban al niño $(73,9 \%)$, respondían por la preparación de los alimentos de lunes a viernes $(76,8 \%)$, el sábado $(71,4 \%)$ y el domingo $(67,9 \%)$, decidían la alimentación de lunes a viernes $(69,6 \%)$, el sábado $(64,3 \%)$ y el domingo (61,6\%). Por otra parte, el padre fue quien principalmente aportó al sustento del hogar (36,6\%), aunque esta responsabilidad fue compartida entre padre y madre en 30,4\% de los hogares. La decisión respecto a la educación de los hijos y el acompañamiento del niño mientras consume alimentos fue compartida para $58,0 \%$ y $41,1 \%$, respectivamente (tabla 4).

En el análisis multivariado en toda la muestra se encontró un aumento significativo del promedio del puntaje $z$ para la edad y sexo con sexo del menor (masculino: $\beta=0,21)$. Así el promedio del puntaje $z$ del IMC para edad y sexo aumentó significativamente 0,85 cuando la abuela era quien respondía por la preparación de los alimentos de lunes a viernes comparado con la madre; 0,77 cuando otro familiar, vecina o amiga decidía la alimentación de lunes a viernes, y 0,57 cuando el preescolar era quien tomaba esa decisión compa-

TABLA 3

Características socioeconómicas de los hogares de los niños y niñas de la Fundación Colombo-Alemana asociadas al puntaje z IMC para edad y sexo. Floridablanca, Colombia, 2013.

\begin{tabular}{|c|c|c|c|c|c|c|c|c|c|c|c|}
\hline \multirow[t]{2}{*}{ Variable } & \multicolumn{2}{|c|}{$\begin{array}{l}\text { Medida de } \\
\text { resumen }^{1}\end{array}$} & \multicolumn{2}{|c|}{ IC95\% } & \multicolumn{3}{|c|}{$\begin{array}{l}\text { Puntaje z del IM C } \\
\text { para edad y sexo }\end{array}$} & \multicolumn{4}{|c|}{$\begin{array}{l}\text { Regresión lineal } \\
\text { generalizada }\end{array}$} \\
\hline & & & & & Mediana & P25 & P75 & Coeficiente & & & Valor $p$ \\
\hline $\begin{array}{l}\text { Tasa de ocupación } \\
\text { Estrato socioeconómico }\end{array}$ & 0,66 & 0,26 & 0,61 & $0,71^{2}$ & - & - & - & 0,14 & $-0,18$ & 0,45 & 0,406 \\
\hline $\begin{array}{l}\text { Estrato cero } \\
\text { Estrato } 1 \text { y } 2\end{array}$ & $\begin{array}{l}32 \\
80\end{array}$ & $\begin{array}{l}28,6 \\
71,4\end{array}$ & $\begin{array}{l}20,2 \\
63,1\end{array}$ & $\begin{array}{l}36,9 \\
79,8\end{array}$ & $\begin{array}{l}0,76 \\
0,27\end{array}$ & $\begin{array}{l}0,16 \\
-0,22\end{array}$ & $\begin{array}{l}1,04 \\
0,81\end{array}$ & $-0,38$ & $-0,56$ & $-0,20$ & 0,000 \\
\hline $\begin{array}{c}\text { Ingresos familiares } \\
0-0,5 \mathrm{SMLV} \\
0,6-1,0 \mathrm{SMLV} \\
1,1-1,5 \mathrm{SMLV} \\
1,5-3,9 \mathrm{SMLV}\end{array}$ & $\begin{array}{l}14 \\
38 \\
28 \\
32\end{array}$ & $\begin{array}{l}12,5 \\
33,9 \\
25,0 \\
28,6\end{array}$ & $\begin{array}{l}6,4 \\
25,2 \\
17,0 \\
20,2\end{array}$ & $\begin{array}{l}18,6 \\
42,7 \\
33,0 \\
36,9\end{array}$ & $\begin{array}{l}0,72 \\
0,42 \\
0,69 \\
0,20\end{array}$ & $\begin{array}{l}0,13 \\
-0,46 \\
0,07 \\
-0,21\end{array}$ & $\begin{array}{l}0,92 \\
0,80 \\
1,05 \\
0,74\end{array}$ & $\begin{array}{l}-0,46 \\
-0,04 \\
-0,42\end{array}$ & $\begin{array}{l}-0,73 \\
-0,32 \\
-0,70\end{array}$ & $\begin{array}{l}-0,19 \\
0,25 \\
-0,14\end{array}$ & $\begin{array}{l}0,001 \\
0,806 \\
0,003\end{array}$ \\
\hline $\begin{array}{l}\text { Procedencia } \\
\text { Urbana } \\
\text { Rural }\end{array}$ & $\begin{array}{l}74 \\
32\end{array}$ & $\begin{array}{l}69,8 \\
30,2\end{array}$ & $\begin{array}{l}57,3 \\
20,2\end{array}$ & $\begin{array}{l}74,8 \\
36,9\end{array}$ & $\begin{array}{l}0,46 \\
0,37\end{array}$ & $\begin{array}{l}-0,09 \\
-0,35\end{array}$ & $\begin{array}{l}0,91 \\
0,76\end{array}$ & $-0,24$ & $-0,43$ & $-0,05$ & 0,012 \\
\hline $\begin{array}{l}\text { Familia es desplazada } \\
\mathrm{No} \\
\mathrm{Si}\end{array}$ & $\begin{array}{l}62 \\
46\end{array}$ & $\begin{array}{l}57,4 \\
42,6\end{array}$ & $\begin{array}{l}46,2 \\
32,0\end{array}$ & $\begin{array}{l}64,6 \\
50,2\end{array}$ & $\begin{array}{l}0,43 \\
0,37\end{array}$ & $\begin{array}{l}-0,14 \\
-0,26\end{array}$ & $\begin{array}{l}0,91 \\
0,81\end{array}$ & $-0,10$ & $-0,28$ & 0,07 & 0,241 \\
\hline $\begin{array}{l}\text { Recibe subsidios gubern } \\
\text { No } \\
\mathrm{Si}\end{array}$ & $\begin{array}{l}\text { menta } \\
41 \\
71\end{array}$ & $\begin{array}{l}\text { de ON } \\
36,6 \\
63,4\end{array}$ & $\begin{array}{l}\text { amigos } \\
27,7 \\
54,5\end{array}$ & $\begin{array}{l}\text { ira el so } \\
45,5 \\
72,3\end{array}$ & $\begin{array}{l}\text { enimiento } \\
0,25 \\
0,50\end{array}$ & $\begin{array}{l}-0,14 \\
-0,25\end{array}$ & $\begin{array}{l}0,88 \\
0,91\end{array}$ & 0,07 & $-0,10$ & 0,24 & 0,418 \\
\hline $\begin{array}{l}\text { Tenencia de vivienda } \\
\text { Propia } \\
\text { Arrendada } \\
\text { Invasion }\end{array}$ & $\begin{array}{l}36 \\
47 \\
29\end{array}$ & $\begin{array}{l}32,1 \\
42,0 \\
25,9\end{array}$ & $\begin{array}{l}23,5 \\
32,8 \\
17,8\end{array}$ & $\begin{array}{l}40,8 \\
51,1 \\
34,0\end{array}$ & $\begin{array}{l}0,37 \\
0,13 \\
0,58\end{array}$ & $\begin{array}{l}-0,07 \\
-0,36 \\
0,22\end{array}$ & $\begin{array}{l}0,86 \\
0,88 \\
0,91\end{array}$ & $\begin{array}{l}-0,32 \\
0,04\end{array}$ & $\begin{array}{l}-0,52 \\
-0,17\end{array}$ & $\begin{array}{l}-0,13 \\
0,26\end{array}$ & $\begin{array}{l}0,001 \\
0,705\end{array}$ \\
\hline $\begin{array}{l}\text { Número de personas que } \\
1-4 \\
5-9\end{array}$ & $\begin{array}{l}\text { confor } \\
58 \\
49\end{array}$ & $\begin{array}{l}\text { an el nú } \\
54,2 \\
45,8\end{array}$ & $\begin{array}{l}\text { familia } \\
42,5 \\
34,6\end{array}$ & $\begin{array}{l}\text { incluido } \\
61,0 \\
52,9\end{array}$ & $\begin{array}{c}\text { el niño } \\
0,46 \\
0,27\end{array}$ & $\begin{array}{l}-0,22 \\
-0,13\end{array}$ & $\begin{array}{l}0,91 \\
0,88\end{array}$ & 0,02 & $-0,16$ & 0,19 & 0,859 \\
\hline $\begin{array}{l}\text { Número de personas que } \\
1-3 \\
4-9\end{array}$ & $\begin{array}{l}\text { vive c } \\
52 \\
60\end{array}$ & $\begin{array}{l}\text { el niño } \\
46,4 \\
53,6\end{array}$ & $\begin{array}{l}37,2 \\
44,3\end{array}$ & $\begin{array}{l}55,7 \\
62,8\end{array}$ & $\begin{array}{l}0,50 \\
0,27\end{array}$ & $\begin{array}{l}-0,16 \\
-0,16\end{array}$ & $\begin{array}{l}0,91 \\
0,84\end{array}$ & $-0,01$ & $-0,18$ & 0,16 & 0,903 \\
\hline $\begin{array}{l}\text { Número de personas que } \\
0-1 \\
2-4\end{array}$ & $\begin{array}{l}\text { duerm } \\
60 \\
52\end{array}$ & $\begin{array}{l}\text { con el } \\
53,6 \\
46,4\end{array}$ & $\begin{array}{l}\text { en el r } \\
44,3 \\
37,2\end{array}$ & $\begin{array}{l}\text { smo cua } \\
62,8 \\
55,7\end{array}$ & $\begin{array}{r}\text { rto } \\
0,37 \\
0,43\end{array}$ & $\begin{array}{l}-0,19 \\
-0,14\end{array}$ & $\begin{array}{l}0,91 \\
0,91\end{array}$ & 0,07 & $-0,10$ & 0,23 & 0,428 \\
\hline $\begin{array}{l}\text { Número de niños menor } \\
\qquad 2-4\end{array}$ & $\begin{array}{l}\text { s de } 7 \\
1 \\
52\end{array}$ & $\begin{array}{l}\text { os en e } \\
60 \\
46,4\end{array}$ & $\begin{array}{l}\text { ar } \\
53,6 \\
37,2\end{array}$ & $\begin{array}{l}44,3 \\
55,7\end{array}$ & $\begin{array}{l}62,8 \\
0,24\end{array}$ & $\begin{array}{l}0,46 \\
-0,26\end{array}$ & $\begin{array}{l}-0,12 \\
0,79\end{array}$ & $\begin{array}{l}0,94 \\
-0,24\end{array}$ & $-0,40$ & $-0,07$ & 0,005 \\
\hline
\end{tabular}

IMC= Indice de Masa Corporal. P25= Percentil 25. P75= Percentil 75. IC95\% Intervalo de confianza del 95\%. SMVL: Salarios mínimos legales vigentes. - No aplica para variables cuantitativas.

1 Promedio y desviación estándar para la variable Tasa de ocupación, frecuencia absoluta (n) y relativa (\%) en las demás variables.

2 Intervalo de confianza del 95\% para el promedio. 
rado con la decisión tomada por la madre. Además, aumentó significativamente en promedio 0,67 cuando el menor comía solo en comparación con comer con ambos padres (tabla 5).
El promedio del puntaje z disminuyó 0,02 por cada año que aumentaba la edad de las madres. Así mismo, disminuyó en promedio 0,62 en los hijos de madres sin compa-

TABLA 4

Responsables del cuidado, alimentación, educación y sostenimiento económico de los hogares de los niños y niñas de la Fundación Colombo-Alemana asociadas al puntaje z IMC para edad y sexo. Floridablanca, Colombia, 2013.

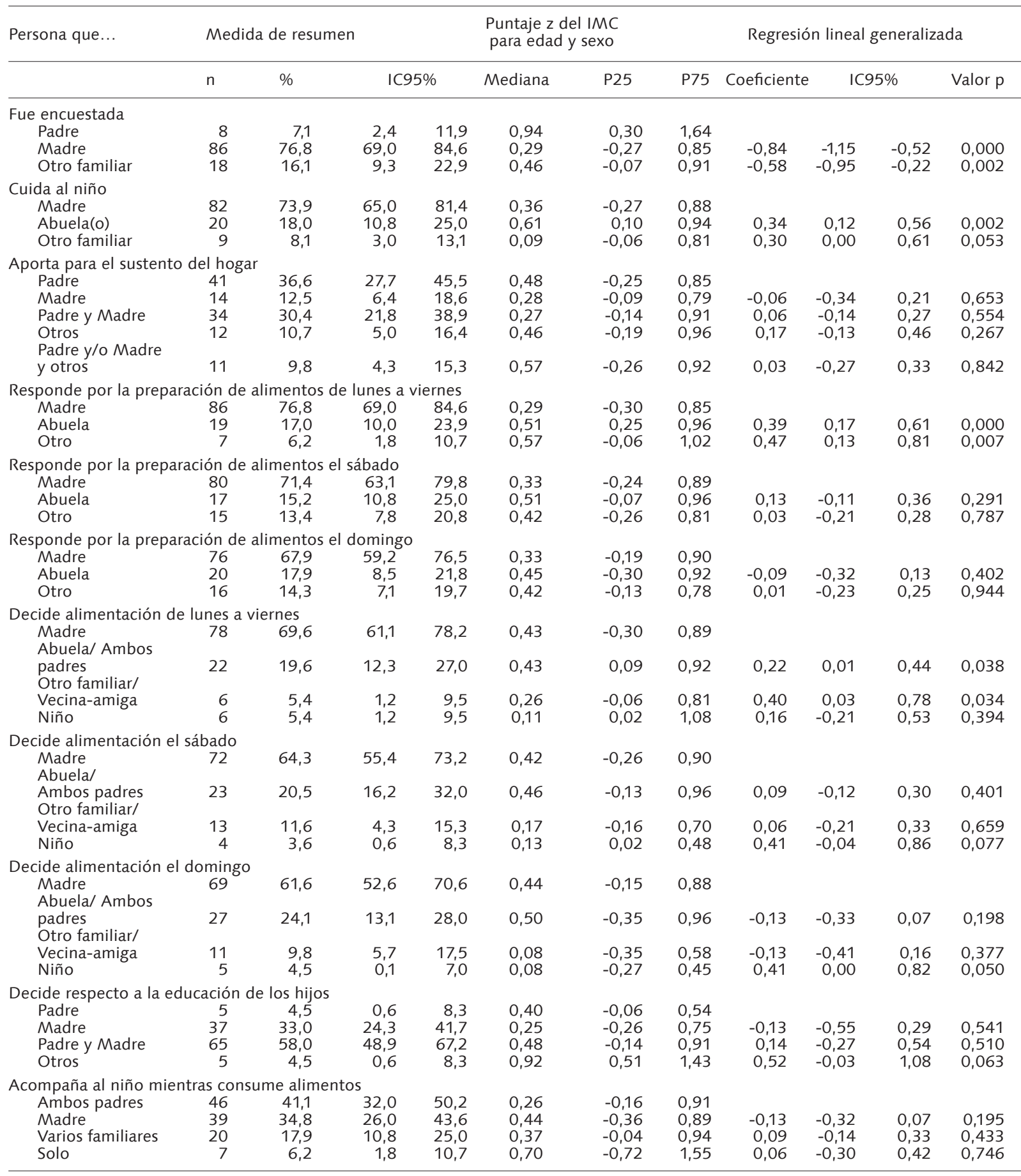


ñero permanente comparado con aquellas en unión libre o casadas; 0,59 en hogares de estratos 1 y 2 comparado con estrato cero; 0,76 en hogares con ingresos familiares entre 0,6 y 1,0 SMLV y 0,70 en hogares con ingresos entre 1,5 y 3,9 SMLV comparado con hogares con ingresos entre $0 \mathrm{y}$ $0,5 \mathrm{SMLV} ; 0,27$ en hogares de procedencia rural comparado con urbana; 0,22 en hogares que tenían entre 2 y 4 niños menores de 7 años comparado con hogares con un solo niño menor de 7 años; y 0,53 cuando el niño consumía alimentos en compañía de varios familiares comparado con la compañía de ambos padres (tabla 5).

En el análisis por sexo se encontró que el IMC de los niños varones se asoció con la procedencia rural de la familia, la preparación de alimentos por la abuela, la ausencia de compañía en las comidas y cuando la madre no vive con un compañero. En las niñas, la edad de la madre, la compañía durante la comida por la madre u otro familiar y cuando la decisión de la alimentación es por otro familiar o la misma niña (tabla 5).

\section{DISCUSIÓN}

Factores económicos. En la muestra global, en los varones y en las niñas, se encontró una asociación inversa entre el estrato socio económico (ESE), los ingresos familiares y el IMC para edad y sexo, ajustado por la edad y el estado civil de la madre, la procedencia de la familia y el número de niños menores de 7 años en el hogar.

El IMC fue menor en preescolares de estrato 1 y 2 comparado con los de estrato cero. Resultados similares se reportaron en una revisión sistemática de 34 estudios publicados en los últimos 15 años (7), en niños escolares de massachusetts (19) y en preescolares libaneses (8). El exceso

\section{TABLA 5}

Factores asociados al puntaje z del IMC para edad y sexo en los niños y niñas de la Fundación Colombo-Alemana de Floridablanca, Colombia (2013). Análisis multivariado usando modelo lineal generalizado múltiple.

\begin{tabular}{|c|c|c|c|c|c|c|c|c|c|c|c|c|}
\hline \multirow[b]{2}{*}{ Variable } & \multicolumn{4}{|c|}{ Global = 100} & \multicolumn{4}{|c|}{ Niñas $=49$} & \multicolumn{4}{|c|}{ Niños $=51$} \\
\hline & $\begin{array}{l}\text { Coefi } \\
\text { ciente } \\
\text { Beta }\end{array}$ & \multicolumn{2}{|l|}{ IC95\% } & Valor $\mathrm{p}$ & $\begin{array}{l}\text { Coefi } \\
\text { ciente } \\
\text { Beta }\end{array}$ & IC95\% & & Valor $\mathrm{p}$ & $\begin{array}{c}\text { Coe } \\
\text { ficiente } \\
\text { Beta }\end{array}$ & IC95\% & & Valor $\mathrm{p}$ \\
\hline \multicolumn{13}{|l|}{ Sexo } \\
\hline Masculino & 0,21 & 0,03 & 0,38 & 0,019 & - & - & - & - & - & - & _- & _ \\
\hline Edad de la madre (años) & $-0,02$ & $-0,04$ & $-0,01$ & 0,000 & $-0,03$ & $-0,04$ & $-0,01$ & 0,001 & 0,00 & $-0,03$ & 0,03 & 0,780 \\
\hline \multicolumn{13}{|l|}{$\begin{array}{l}\text { Estado civil de la madre } \\
\text { Union libre/casada }\end{array}$} \\
\hline $\begin{array}{l}\text { Separada/Divorciada/ } \\
\text { viuda/Madre Soltera } \\
\text { Estrato socioeconómico } \\
\text { Estrato cero }\end{array}$ & $-0,62$ & $-0,83$ & $-0,41$ & 0,000 & $-0,25$ & $-0,53$ & 0,03 & 0,082 & $-1,15$ & $-1,54$ & $-0,77$ & 0,000 \\
\hline \multicolumn{13}{|l|}{$\begin{array}{l}\text { Ingresos familiares } \\
0-0,5 \text { SMLV }\end{array}$} \\
\hline $0,6-1,0$ SMLV & $-0,76$ & $-1,05$ & $-0,47$ & 0,000 & $-0,89$ & $-1,29$ & $-0,49$ & 0,000 & $-0,60$ & $-1,08$ & $-0,11$ & 0,016 \\
\hline $1,1-1,5$ SMLV & $-0,15$ & $-0,46$ & 0,16 & 0,332 & $-0,33$ & $-0,79$ & 0,13 & 0,156 & $-0,19$ & $-0,74$ & 0,36 & 0,499 \\
\hline $1,5-3,9$ SMLV & $-0,70$ & $-1,01$ & $-0,38$ & 0,000 & $-0,68$ & $-1,14$ & $-0,23$ & 0,003 & $-0,80$ & $-1,31$ & $-0,29$ & 0,002 \\
\hline \multicolumn{13}{|l|}{ Procedencia } \\
\hline Rural & $-0,27$ & $-0,45$ & $-0,08$ & 0,005 & $-0,21$ & $-0,45$ & 0,04 & 0,100 & $-0,54$ & $-0,89$ & $-0,19$ & 0,002 \\
\hline \multicolumn{13}{|c|}{ Número de niños menores de 7 años en el hogar } \\
\hline \multicolumn{13}{|l|}{1} \\
\hline \multicolumn{13}{|c|}{ Responde por la preparación de alimentos de lunes a viernes } \\
\hline \multicolumn{13}{|l|}{ Madre } \\
\hline Abuela & 0,85 & 0,49 & 1,21 & 0,000 & 0,28 & $-0,27$ & 0,83 & 0,316 & 1,43 & 0,83 & 2,02 & 0,000 \\
\hline Otro & 0,26 & $-0,30$ & 0,82 & 0,363 & $-0,11$ & $-1,21$ & 0,99 & 0,850 & 0,79 & $-0,08$ & 1,66 & 0,077 \\
\hline \multicolumn{13}{|c|}{ Decide alimentación de lunes a viernes } \\
\hline \multicolumn{13}{|l|}{ Madre } \\
\hline Abuela/ Ambos padres & $-0,02$ & $-0,31$ & 0,27 & 0,894 & 0,21 & $-0,22$ & 0,64 & 0,339 & $-0,35$ & $-0,84$ & 0,14 & 0,161 \\
\hline Otro familiar/Vecina-amiga & 0,77 & 0,18 & 1,35 & 0,010 & 1,02 & 0,27 & 1,78 & 0,008 & 0,14 & $-1,01$ & 1,28 & 0,817 \\
\hline Niño & 0,57 & 0,13 & 1,00 & 0,010 & 0,44 & 0,01 & 0,87 & 0,045 & 0,95 & $-0,16$ & 2,06 & 0,092 \\
\hline \multicolumn{13}{|c|}{ Acompaña al niño mientras consume alimentos } \\
\hline Ambos padres & & & & & & & & & & & & \\
\hline Madre & $-0,12$ & $-0,31$ & 0,07 & 0,204 & $-0,31$ & $-0,59$ & $-0,02$ & 0,035 & 0,22 & $-0,11$ & 0,55 & 0,189 \\
\hline Varios familiares & $-0,53$ & $-0,83$ & $-0,24$ & 0,000 & $-0,51$ & $-0,90$ & $-0,12$ & 0,010 & $-0,04$ & $-0,66$ & 0,58 & 0,905 \\
\hline Solo & 0,67 & 0,30 & 1,05 & 0,000 & 0,37 & $-0,09$ & 0,82 & 0,112 & 0,89 & 0,23 & 1,56 & 0,009 \\
\hline Linktest & 0,800 & & & & 0,864 & & & & 0,519 & & & \\
\hline
\end{tabular}

IC 95\% Intervalo de confianza del 95\%. SMLV: Salario mínimo legal vigente. - No aplica. 
de peso se ha incrementado en mayor medida en zonas de pobreza $(1,2)$, cabe mencionar que la población estudiada se encontraba en situación de vulnerabilidad, pertenecían a estratos socioeconómicos bajos o estaban en situación de desplazamiento, y en estos grupos la alimentación se caracteriza principalmente por alimentos económicos y altamente energéticos (20-22).

Factores sociales. El sexo de los preescolares se asoció a un mayor IMC, fueron los niños varones quienes presentaron los IMC más altos, similar a los resultados de la ENSIN (5) y contrario a lo observado en población colombiana de 5 a 17 años (23).

Se encontró relación inversa y significativa entre la edad de la madre y el IMC tanto de la muestra global, como de las niñas. Una madre con más edad y experiencia tiene la posibilidad de contar con más información sobre los cuidados del hijo, al respecto Moreno y Cols., afirman que el cuidado del menor se ve influenciado por la información que haya recibido el cuidador (24).

La procedencia rural de la familia se asoció a un menor IMC en la muestra global y en los niños varones. La ENSIN 2010, reportó en el área urbana mayor prevalencia de exceso de peso en el grupo de 5 a 17 años (19,2\% vs 13,4\%), pero no en los menores de 5 años (5).

Sí la familia tenía entre 2 y 4 menores de 7 años, los preescolares presentaron menor IMC. Otros estudios han reportado asociación entre el número de menores de 7 años (25), el número de integrantes de la familia, o número de hermanos con déficit nutricional $(26,27)$.

Cuando las madres no vivían con un compañero los hijos presentaron menor IMC (muestra global y varones), es probable que la ausencia del aporte económico del padre, incida en una menor disponibilidad y consumo de alimentos y por tanto en un menor IMC. Otras investigaciones evidenciaron un mayor IMC cuando los hijos viven con sus dos padres, los factores asociados fueron, el doble ingreso de la familia, el contar con un empleo y el tiempo de permanencia en él (28). En familias víctimas del conflicto armando por desplazamiento, la compra de alimentos está asociada al número de miembros de la familia que trabajaban y aportan (20).

La ausencia de compañía se asoció con un mayor IMC (muestra total y varones), mientras que la compañía de otros familiares o sólo de la madre con un IMC menor (muestra total y niñas), teniendo como referencia el acompañamiento de los dos padres, resultados similares fueron reportados en otros estudios $(9,29,30)$.

La preparación de alimentos a cargo de la abuela, se asoció a un mayor IMC (muestra global total y varones), en la localidad se evidenció un menor IMC en preescolares cuyas madres eran las responsables de preparar los alimentos (9). Se han obtenido resultados contradictorios sobre la participación de los abuelos en la alimentación, algunos mencionan efectos negativos (31), otros positivos $(24,32,33)$. Moreno y Cols., destacan los cambios de roles al interior de la familia y la importancia de la abuela en el cuidado de los nietos (24).

La decisión respecto a la alimentación, se asoció a un mayor IMC cuando estaba a cargo de otro familiar distinto a la madre, o cuando era realizada por la niña (muestra total y niñas). Resultados similares se reportaron en el estudio de Geoffroy y Cols., quienes además destacan la influencia positiva de la madre en la alimentación familiar y en el estado nutricional de los hijos (34).

La limitación de este estudio consiste en la tasa de no respuesta de algunas variables, por lo cual el análisis multi- variado se redujo a una muestra de 100 niños(as), esto puede explicarse porque algunas preguntas hacían referencia a aspectos particulares del padre y dado que algunas familias eran monoparentales era difícil recopilar estos datos.

En conclusión, el IMC en los preescolares estudiados estuvo asociado de manera inversa con el estrato socioeconómico y los ingresos familiares. Otras variables asociadas a un mayor IMC fueron, la preparación de alimentos a cargo de la abuela, la decisión de la alimentación por otro familiar o por el mismo preescolar y la ausencia de compañía durante el momento del consumo.

Un IMC menor estuvo asociado a la edad de la madre, madre sin compañero, procedencia rural de la familia, número de menores de 7 años, acompañamiento durante el consumo de alimentos por familiares y no por los dos padres.

Como recomendación, las políticas para prevenir la obesidad deben considerar la población infantil de bajos ingresos, grupo que ha mostrado ser el más indicado para implementar estrategias de prevención $(4,35)$. Es deseable involucrar a las abuelas y a otros cuidadores. Como estrategias, la educación nutricional es recomendable por la eficacia en la reducción del IMC de niños y adolescentes (36).

La relevancia de este trabajo radica en que existen pocas investigaciones en el ámbito nacional que hayan abordado los factores socioeconómicos asociados al IMC en preescolares de bajos ingresos y que discriminen los factores asociados al IMC de los varones y las niñas.

\section{RESUMEN}

El objetivo del presente estudio fue determinar la asociación entre factores socioeconómicos y el puntaje z del Índice de Masa Corporal (IMCZ) en preescolares de la Fundación Colombo-Alemana de Floridablanca, Colombia. Una muestra aleatoria de 112 preescolares de 2 a 5 años fue medida y pesada; sus padres o cuidadores respondieron una encuesta. Los modelos lineales generalizados múltiples mostraron que en toda la muestra el IMCZ disminuyó significativamente en estratos socioeconómicos 1 y 2 comparado con estrato cero y en familias con ingresos altos. En niñas, aumentó significativamente cuando otro familiar, vecina o amiga decidía la alimentación de lunes a viernes y cuando la niña decidía. En niños, aumentó cuando comían solos y cuando la abuela era responsable de preparar los alimentos de lunes a viernes. Se concluye que los factores socioeconómicos que influyen sobre el IMCZ son similares a los que influyen en la inequidad en salud.

Palabras clave: Factores socioeconómicos, factores de riesgo, indice de masa corporal, obesidad pediátrica.

\section{BIBLIOGRAFIA}

1. Popkin $B M$. An overview on the nutrition transition and its health implications:the Bellagio meeting. Public Health Nutr. 2002; 5(1A):93-103.

2. Popkin BM, Adair LS, Ng SW. Global Nutrition Transition: The Pandemic of Obesity in Developing Countries. Nutr Rev. 2012; 70(1):3-21.

3. $\mathrm{Ng} M$, Fleming $T$, Robinson $M$, Thomson $B$, Graetz $N$, Zhu $S$, et al. Global, regional, and national prevalence of overweight and obesity in children and adults during 1980-2013: a systematic analysis for the Global Burden of Disease Study 2013. The Lancet 2014;384(9945):766-81.

4. Organización Panamericana de la Salud. Organización Mundial de la Salud. 154.a Sesión del comité ejecutivo. 
Plan de acción para la prevención de la obesidad en la niñez y la adolescencia. Washington. Junio de 2014. CE154/16.

5. Instituto Colombiano de Bienestar Familiar. Encuesta Nacional de la Situación Nutricional en Colombia-ENSIN 2010 -. Bogotá. 2011.

6. Duelo M, Escribano E, Muñoz F. Obesidad. Rev Pediatr Aten Primaria 2009;2 (Supl 16):s127-42.

7. Shrewsbury V, Wardle J. Socioeconomic status and adiposity in childhood: a systematic review of cross-sectional studies 1990-2005. Obesity 2008; 16(2):275-84.

8. Klebanov PK, Evans GW, Brooks-Gunn J. Poverty, ethnicity, and risk of obesity among low birth weight infants. J Appl Develop Psychol 2014; 35(3):245-53.

9. Prada GE, Gutierrez $M$, Sánchez $X L$, Rueda LP, Angarita A. Asociación entre factores ambientales y pautas de crianza con el Índice de Masa Corporal de preescolares de hogares infantiles de Floridablanca, Colombia. Rev Chil Nutr. 2014; 41(3): 284-91.

10. Valdés J, Royo-Bordonada MA. Prevalence of childhood obesity in Spain. National Health Survey 2006-2007. Nutr Hosp. 2012; 27(1):154-60.

11. McDonald CM, Baylin A, Arsenault JE, Mora-Plazas $M$, Villamor $E$. Overweight is more prevalent than stunting and is associated with socioeconomic status, maternal obesity, and a snacking dietary pattern in school children from Bogota, Colombia. J Nutr. 2009; 139(2):370-6.

12. StataCorp. Stata Statistical Software: Release 13. Texas, United States of America: StataCorp LP; 2013.

13. Stewart A, Marfell-Jones M, Olds T, de Ridder H. International standards for anthropometric assessment. Lower Hutt, New Zealand: ISAK; 2011.

14. Organización Mundial de la Salud. Software ANTHRO 3.1. Ginebra, Suiza.: OMS; 2010.

15. Fonseca Mde J, Andreozzi VL, Faerstein E, Chor D, Carvalho MS. Alternatives in modeling of body mass index as a continuous response variable and relevance of residual analysis. Cad Saude Publica. 2008; 24(2):473-8.

16. Lauritsen JM, Bruus $M$, Myatt MA. An extended tool for validated dataentry and documentation of data. Odense, Denmark: The EpiData Association; 2001.

17. StataCorp. Stata Statistical Software: Release 13. Texas, United States of America: StataCorp LP; 2013.

18. Resolución 008430 del 4 de octubre de 1993. Por la cual se establecen las normas científicas, técnicas y administrativas para la investigación en salud. 008430 (1993).

19. Eagle TF, Sheetz A, Gurm R, Woodward AC, Kline-Rogers $\mathrm{E}$, Leibowitz R. Understanding childhood obesity in America: Linkages between household income, community resources, and children's behaviors. Am Heart J. 2012; 163:836-43.

20. Prada GE, Herrán OF, Ortiz R. Patrón alimentario y acceso a los alimentos en familias desplazadas en el municipio de Girón, Santander, Colombia. Rev Panam Salud Pública 2008; 23(4):257-63.

21. OPS, WFP. Comisión Europea de ayuda alimentaria, OPS. Estado nutricional, de alimentación y condiciones de salud de la población desplazada por la violencia en seis subregiones del país. Informe final. Bogotá, Colombia.
Diciembre de 2005. http://documents.wfp.org/stellent/ groups/public/documents/liaison_offices/wfp086486. pdf. Consultado en marzo de 2015.

22. Gamboa EM, López N, Vera LM, Prada GE. Patrón alimentario y estado nutricional en niños desplazados en Piedecuesta, Colombia. Rev Salud Pública 2007; 1:12939.

23. Ocampo PR, Prada GE, Herrán OF. Patrones de consumo alimentario y exceso de peso infantil; encuesta de la situación nutricional en Colombia, 2010. Rev Chil Nutr. 2014; 41 (4): 351-9.

24. Moreno-Zavaleta MT, Granada-Echeverri P. Interacciones vinculares en el sistema de cuidado infantil. Rev Latinoam Cienc Soc Niñez Juv. 2014; 12(1):121-39.

25. Herrán Falla OF, Prada Gómez GE, Patiño Benavides GA. ¿Por Qué La Malnutrición En Menores De Doce Años De Bucaramanga?. Salud UIS 2001; 33(1):50-6.

26. Londoño A, Shirley Mejía S. Factores de riesgo para malnutrición relacionados con conocimientos y prácticas de alimentación en preescolares de estrato bajo en Calarcá. 2006-2007. Rev Gerenc Polit Salud 2009; 8(15):77-90.

27. Alcaraz Gloria, Bernal Carlos, Cornejo William, Figueroa Natalia, Múnera Margarita. Estado nutricional y condiciones de vida de los niños menores de cinco años del área urbana del municipio de Turbo, Antioquia, Colombia, 2004. Biomédica 2008; 28(1): 87-96.

28. Morrissey TW. Trajectories of growth in body mass index across childhood: Associations with maternal and paternal employment. Social Sci Med. 2013; 95:60-8.

29. Chacón A. Control y presión al comer en madres de preescolares costarricenses, y su relación con la clase social, la escolaridad, las prácticas de alimentación temprana y el índice de masa corporal de sus hijos e hijas. Pobl Salud Mesoam 2011; 9(1): Disponible en http://revistas.ucr. ac.cr/index.php/psm/article/view/728/791. Consultado en marzo de 2014.

30. Hoerr SL, Hughes SO, Fisher JO, Nicklas TA, Liu Y, Shewchuk RM. Associations among parental feeding styles and children's food intake in families with limited incomes. Int J Behav Nutr Phys Act 2009;6:55. Disponible en http:// ncbi.nlm.nih.gov/pmc/articles/PMC2739505/ pdf/. Consultado en Febrero de 2015.

31. Farrow C. A comparison between the feeding practices of parents and grandparents. Eating Behav. 2014; 15:339-42.

32. Fouts HN, Brookshire RA. Who feeds children? A child'seye-view of caregiver feeding patterns among the Aka foragers in Congo. Social Sci Med. 2009; 69(2):285-92.

33. Reid J, Schmied V, Beale B. 'I only give advice if I am asked': Examining the grandmother's potential to influence infant feeding decisions and parenting practices of new mothers. Women Birth 2010;23:74-80.

34. Geoffroy MC, Power C, Touchette E, Dubois I, Boivin $M$, Séguin JR, Tremblay RE, Coté SM. Childcare and Overweight or Obesity over 10 Years of Follow-Up. J Pediatr. 2013; 162 (4):753-8.

35. Moraga F, Rebollo MJ, Bórquez P, Cáceres J, Castillo C. Tratamiento de la obesidad infantil: Factores pronósticos asociados a una respuesta favorable. Rev Chil Pediatr. 2003; 74 (4):374-80. 